\title{
Numerical Study on the Flow Mechanism of Compressor Rotor Blade Vibration under Different Inlet Probe Configurations
}

\author{
Rongfei Yang $\mathbb{D}^{1,2}$ Guoliang Wang $\mathbb{D}^{1,2}$ Haixu Liu, ${ }^{1,2}$ Honghui Xiang, ${ }^{3}$ and Jie Gao ${ }^{3}$ \\ ${ }^{1}$ College of Energy and Power Engineering, Nanjing University of Aeronautics and Astronautics, Nanjing 210016, China \\ ${ }^{2}$ Jiangsu Province Key Laboratory of Aerospace Power System, Nanjing, 210016 Jiangsu, China \\ ${ }^{3}$ AECC Sichuan Gas Turbine Establishment, Mianyang 621000, China
}

Correspondence should be addressed to Rongfei Yang; yrf@nuaa.edu.cn

Received 30 August 2020; Revised 18 October 2020; Accepted 12 November 2020; Published 2 December 2020

Academic Editor: Dakun Sun

Copyright (c) 2020 Rongfei Yang et al. This is an open access article distributed under the Creative Commons Attribution License, which permits unrestricted use, distribution, and reproduction in any medium, provided the original work is properly cited.

In the performance test of a compressor, the overstrain alarm of a rotor blade occurred and it was thought to be caused by a largesized inlet probe. To explain and further avoid the occurrence of this phenomenon, the influences of probe strut configuration on the vibration strain of the compressor rotor blade and the corresponding flow mechanism are studied by using a one-way fluidstructure coupling calculation method. Firstly, the probe strut is simplified as a cylinder with a diameter of $10 \mathrm{~mm}$ and located upstream of the inlet stator with the strut wake impinging on the stator blade according to the compressor test. Then, the three scenarios are considered: moving the strut away from the stator blade in axial direction, shifting the strut half of the stator pitch circumferentially, and reducing the strut diameter. The analysis results show that the characteristics of blade vibration are determined by the excitation force on the rotor blade. Under the interaction between the large-sized strut and the compressor, in addition to the excitation force with the strut passing frequency, a force with a lower frequency, namely, the strut-wakeinduced frequency, is also observed. The amplitude of the excitation force on the rotor blade depends on the probe configuration. When one of the excitation force frequencies is close to a natural frequency of the rotor blade, the blade resonates, and the amplitude of blade strain varies with the amplitude of the excitation force. In order to reduce the adverse effect of upstream strut wake on the compressor rotor blade vibration, the inlet probe strut should be designed with a smaller diameter and be placed further upstream of the stator in such a way that the strut wake vortex passes through the midpassage of the following stator.

\section{Introduction}

The immersed total temperature/total pressure probe is widely used in the aerodynamic performance test of compressors. As a foreign body, the probe obstructs the flow passage and produces wake vortex, resulting in change of the compressor aerodynamic performance and the blade vibration characteristic.

Most of the published researches on the interaction between an inlet probe and compressors focus on aerodynamic performance of compressors. According to the experimental results of Ma et al. [1], the static pressure rise and total pressure rise of low-speed compressor are reduced after installing a large-sized probe strut. The numerical studies by Yang et al. [2] showed that the reduction of 1.5-stage com- pressor performance was related to the diameter and the number, but not the circumferential position of the inlet probe strut. The numerical results of Ma et al. [3] showed that the change of compressor performance came from the interaction between the wake vortices shedding from the probe strut and the vortices generated inside the compressor blade passage. In the compressor experiment conducted by Lepicovsky [4], the outflow pressure and the axial velocity distribution of adjacent rotor blade passages were affected by the inlet probe strut rotating synchronously with the rotor. The experimental results of Xiang et al. [5] showed that the linear cascade loss reached the minimum value when the shedding vortices of the probe strut of $10 \mathrm{~mm}$ in diameter passed through the blade passage rather than impinged on the blade. 


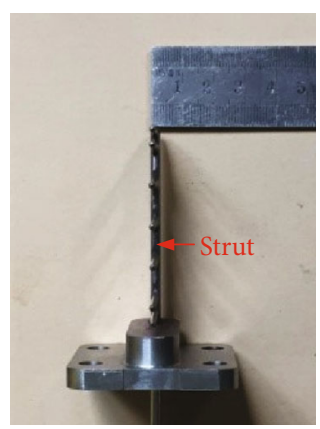

(a)

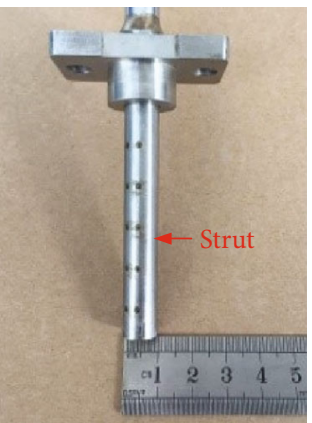

(b)

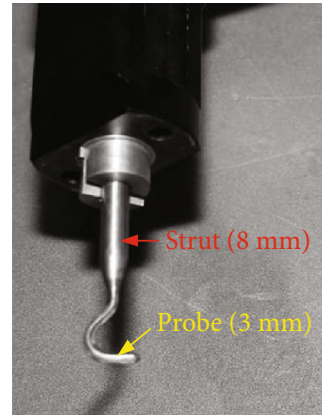

(c)

Figure 1: Photos of the inlet measurement probes: (a) total pressure probe; (b) orientation probe; (c) five-hole orientation probe.

In the studies of probe-compressor interaction mentioned above, the probe is usually simplified as a strut or cylinder. Teruna et al.'s numerical results [6] showed that the blade surface pressure fluctuates greatly when the upstream cylinder wakes impinge on the compressor blade. The blade resonance may occur under the influence of large-scale Kármán vortex street shedding from upstream cylinders $[7,8]$. Besides that, there is no more public literature about the compressor blade vibration caused by the inlet probe wake. Researches on compressor blade vibration mainly focus on self-excited vibration and forced vibration caused by the unsteady flow of the rotor-stator interaction $[9,10]$, tip leakage vortex [11], rotating stall $[12,13]$, and so on. The reasons why people pay little attention to blade vibration caused by the probe strut-compressor interaction are two folds. On the one hand, it is relatively rare to have blade vibration failure caused by a strut-compressor interaction in compressor tests. On the other hand, a potential compressor blade vibration failure may be easily prevented by replacing the original probe strut with a smaller one.

Nevertheless, in the performance test of a compressor with an inlet probe strut of $10 \mathrm{~mm}$ in diameter, it was found that the dynamic strain of a rotor blade exceeded the predefined limit. The strain over limit warning was cleared by removing the probe strut of $10 \mathrm{~mm}$ or replacing it with a small-sized inlet probe strut of $8 \mathrm{~mm}+3 \mathrm{~mm}$ in diameters (seen in Figure 1). In order to avoid the recurrence of the compressor blade vibration problem, it is necessary to analyze the flow mechanism of this phenomenon and further establish some guidelines for designing and placing inlet probe struts, such as the probe diameter and the installation position in compressor performance tests. Since the cost of numerical study is much less than that of experimental study, fluid-structure coupling calculations are carried out to investigate the blade vibration and the flow mechanism of the tested compressor with different scenarios considering the probe diameter and positioning.

\section{Description of the Experimental Results}

The experimental results were taken from a three-stage axial compressor performance test. At the design rotational speed of 7671 RPM, the mass flow, the total pressure ratio, and adiabatic efficiency are $17.9 \mathrm{~kg} / \mathrm{s}, 1.68$, and $85 \%$, respectively.
The hub ratio, the radius at the shroud, the chord at blade tip, and the tip clearance of the first-stage rotor are 0.84 , $328.5 \mathrm{~mm}, 44.5 \mathrm{~mm}$, and $0.5 \mathrm{~mm}$, respectively. Figures $2(\mathrm{a})$ and 2(b) show the axial and circumferential positions of the total pressure probe and the orientation probe mounted on plane 1-1 with a radial inclination of 6.2 degrees. The cylindrical orientation probe was used for measuring the outlet flow angle of upstream preswirl blades (IGV, simulating the last-stage stator blades of a fan). At the midspan section, the probe strut at position (5) is placed at 5.4 times the strut diameter upstream of the leading edge of blade S0 in streamwise and the probe wake just impinges on the leading edge of blade S0 as shown in Figure 2(c). Figure 1 shows the total pressure and the orientation probes used in the compressor performance tests. The cross section of the total pressure probe strut is a long strip of $3 \mathrm{~mm} \times 14 \mathrm{~mm}$ as shown in Figure 1(a), and the orientation probe strut is a cylindrical type. The diameter of the strut in Figure $1(\mathrm{~b})$ is $10 \mathrm{~mm}$, and the strut in Figure 1(c) has a diameter varying from $8 \mathrm{~mm}$ for its top bit to $3 \mathrm{~mm}$ for its tip. Three strain gauges are pasted on the pressure surface of the three blades of the first-stage rotor, respectively, as shown in Figure 3, which are used to monitor the vibration strain signals of the first three natural modes of the rotor blade. The vibration strain measurement system is mainly composed of resistance strain gauge, slip ring wire electrical, Xpod signal conditioner, OROS dynamic data acquisition system, etc. The data acquisition has a sampling frequency of $16.38 \mathrm{kHz}$.

According to the test results, when the compressor speed was greater than 4457 RPM and the inlet orientation probe in Figure 1(b) was used, the strain over limit warning occurred at point $\mathrm{A}$ on the rotor surface. The warning signal disappeared when the orientation probe was removed or replaced with the small-sized probe in Figure 1(c). Some test results of point A are given in Table 1. At 4600 RPM and 5150 RPM, the mass flow rates at the two operating points with the over limit warning are $10.5 \mathrm{~kg} / \mathrm{s}$ and $12.3 \mathrm{~kg} / \mathrm{s}$, respectively. These two points are marked as solid symbol at the computational performance map in Figure 4. The two operating points are away from the choke point and the stall point of the compressor.

In order to understand the cause of blade strain over limit, the modal analysis results of the rotor blade in Table 2 are compared with the frequency analysis results of 


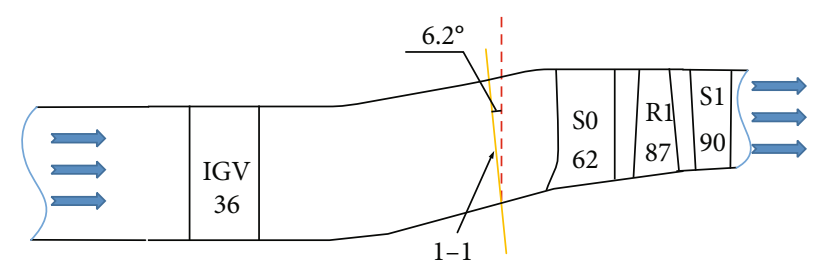

(a)

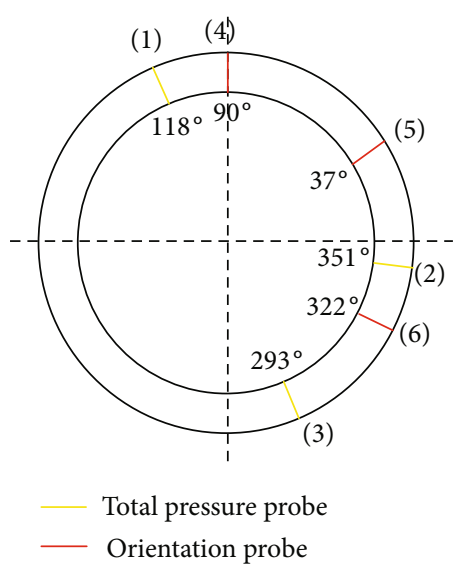

(b)

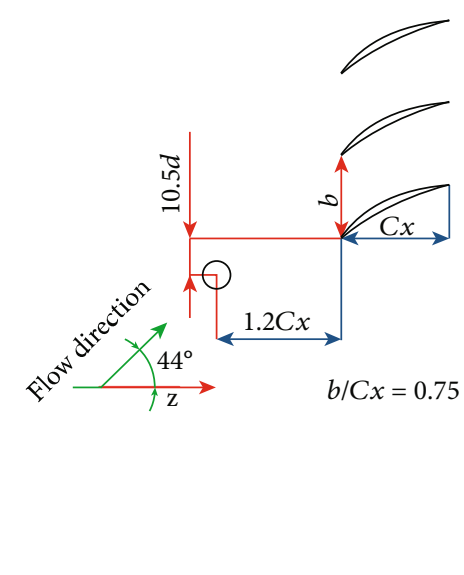

(c)

FIGURE 2: Schematic of the installation position of inlet measurement probe: (a) axial position; (b) circumferential position at plane 1-1; (c) the position of the probe support 5 at $50 \%$ span.

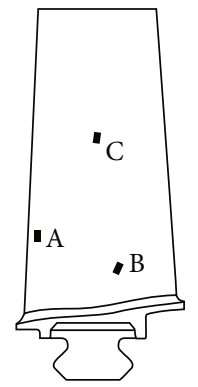

Figure 3: Installed positions of strain gauges on the rotor blade pressure surface.

the measured strain signals in Table 1. The default block Lanczos method of ANSYS software is adopted for modal analysis using the mesh and boundary conditions to be presented in Section 4.2. The blade disk is not considered in the modal analysis, so the calculated natural frequencies of the rotor blade alone are slightly higher than those of the actual rotor [14]. Comparing Table 1 with Table 2, it can be seen that as long as the orientation probe is installed at position (5), the strain amplitude and the maximum strain become very large and the strain dominant frequency at point A has a value close to the first natural mode frequency of the blade. This means that the rotor blade resonance occurs under the influence of the probe at position (5). In Table 1, the blade strain amplitude and the maximum strain for the probe configuration including the orientation probe shown in Figure 1(b) are greater than those for the probe configuration including the five-hole orientation probe shown in
Figure 1(c). This indicates that the vibration amplitude of rotor blade is also related to the probe diameter.

To explain the origin of the excitation force required for the blade resonance, the compressor with an inlet orientation probe (in Figure 1(b)) installed at position (5) is taken as the research object for the fluid-structure coupling calculation in this paper. In addition, in order to prevent the occurrence of the blade resonance and the strain over limit in future compressor tests, the influence of inlet probe strut configuration (the circumferential position, the axial position, and the diameter) on blade vibration is further studied.

\section{Numerical Research Strategy}

Theoretically, the computational domain should be a full annulus of the compressor in Figure 5. However, the computational grid of the full annulus model of the three-stage compressor with an inlet probe will exceed 100 million even if the number of grid points of each blade passage is as low as 300,000 , which is computationally intractable. In order to reduce the size of the computational grid, only the IGV, the orientation probe installed at position (5), the stator row $\mathrm{S} 0$, and the rotor row $\mathrm{R} 1$ are selected. The mesh size is further reduced by setting the computational domain to $1 / 8$ annulus of the compressor of which the blade counts of IGV, strut, S0, and R1 blades are further adjusted from 36:1:62:87 to $32: 8: 64: 88(4: 1: 8: 11)$. As the main body of the orientation probe (Figure 1(b)) is a cylindrical strut, the probe is simplified as a cylindrical strut, abbreviated as "strut" in the following sections. 
TABLE 1: Test results of the strains at point A on the rotor blade.

\begin{tabular}{lccccc}
\hline Strut configuration & $\begin{array}{c}\text { Rotor speed } \\
(\mathrm{RPM})\end{array}$ & $\begin{array}{c}\text { Peak-peak strain amplitude } \\
(\mu \varepsilon)\end{array}$ & $\begin{array}{c}\text { Maximum strain } \\
(\mu \varepsilon)\end{array}$ & $\begin{array}{c}\text { Dominant frequency } \\
(\mathrm{Hz})\end{array}$ & $\begin{array}{c}\text { Strain warning } \\
\text { N }\end{array}$ \\
\hline 1,2 & $4600 / 4900 / 5150$ & $-/ 165 /-$ & $-/ 102 /-$ & $-/-/-$ & No \\
$1,2,5$ (Figure 1(b)) & $4600 / 4900 / 5150$ & $659 / 698 / 810$ & $346 / 372 / 421$ & $852 / 886 / 856$ \\
$1,2,5$ (Figure 1(c)) & $4600 / 4900 / 5150$ & $460 /-/ 643$ & $239 /-/ 362$ & $848 /-/ 865$ & Yes \\
\hline
\end{tabular}

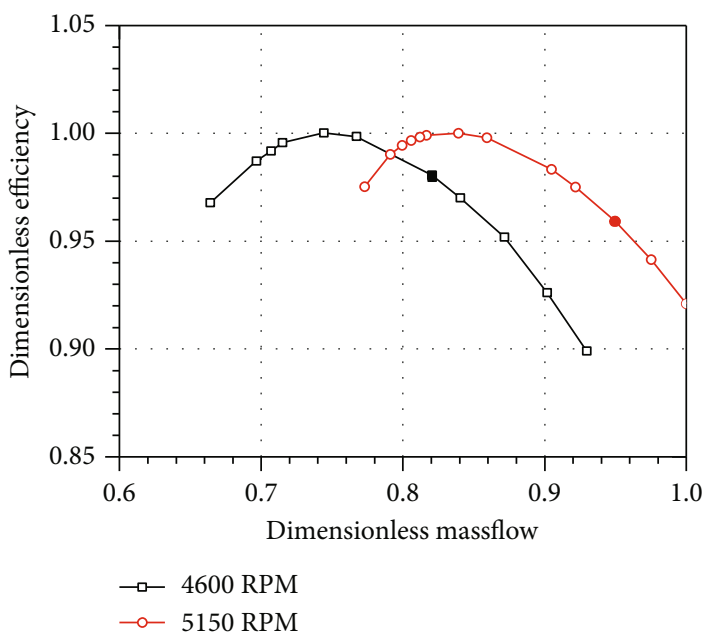

(a)

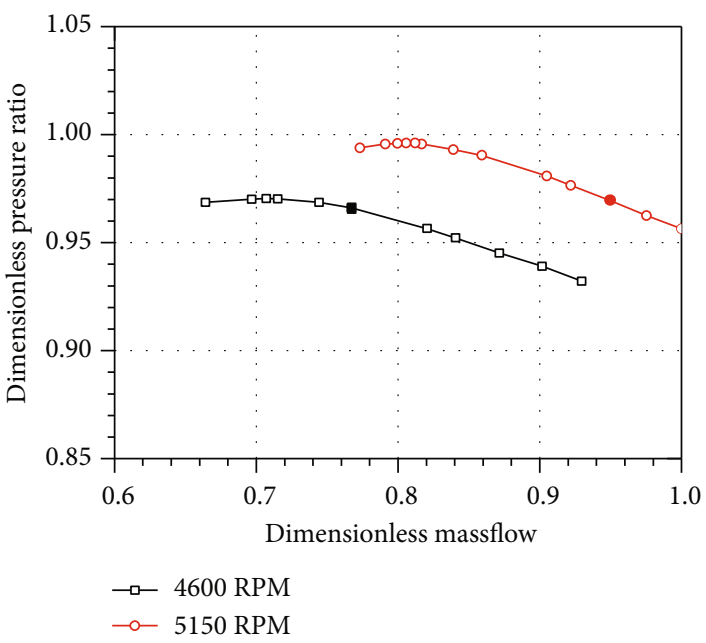

(b)

FiguRE 4: Compressor performance map: (a) dimensionless efficiency and (b) dimensionless pressure ratio.

TABLE 2: Natural frequency of the rotor blade.

\begin{tabular}{lccc}
\hline Modal & 1 & 2 & 3 \\
\hline $4600 \mathrm{RPM}$ & $908 \mathrm{~Hz}$ & $2296 \mathrm{~Hz}$ & $3583 \mathrm{~Hz}$ \\
$4900 \mathrm{RPM}$ & $913 \mathrm{~Hz}$ & $2298 \mathrm{~Hz}$ & $3591 \mathrm{~Hz}$ \\
$5010 \mathrm{RPM}$ & $917 \mathrm{~Hz}$ & $2299 \mathrm{~Hz}$ & $3594 \mathrm{~Hz}$ \\
\hline
\end{tabular}

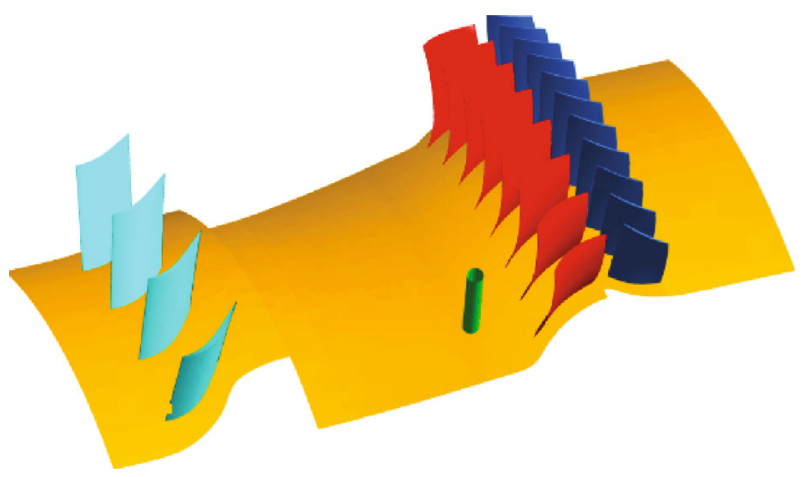

FIGURE 5: Computational model of the compressor with a probe strut.

Although the number of struts increases to 8 , the distance between adjacent struts is $20.6<L / D<25.6$ ( $L$ represents the strut pitch; $D$ is the strut diameter) along blade span, which is much larger than the critical value of 4-5 reported experimentally by Zdravkovich [15]. Thus, the interaction between adjacent struts can be ignored, which means that the vortex
TABLE 3: Four different probe configurations under investigation.

\begin{tabular}{lccc}
\hline Case & Diameter & Axial distance & Circumferential position \\
\hline 1 & $10 \mathrm{~mm}$ & $3.5 \mathrm{R} 1$ & $0 \mathrm{~S}$ \\
2 & $10 \mathrm{~mm}$ & $3.5 \mathrm{R} 1$ & $0.5 \mathrm{~S}$ \\
3 & $10 \mathrm{~mm}$ & $4.5 \mathrm{R} 1$ & $0 \mathrm{~S}$ \\
4 & $5 \mathrm{~mm}$ & $3.5 \mathrm{R} 1$ & $0 \mathrm{~S}$
\end{tabular}

shedding characteristics of one strut and eight struts will be the same. In addition, compared to the real compressor model, the solidities of stator S0 and rotor R1 in the calculation model have been changed by $3.1 \%$ and $1.1 \%$, respectively, which is expected to have negligible impact on the compressor performance. Therefore, the calculation model in Figure 5 can be used to reveal the flow mechanism of the blade vibration induced by the interaction between the strut and the compressor.

At the two operating points of (4600 RPM, $10.5 \mathrm{~kg} / \mathrm{s}$ ) and (5150 RPM, $12.3 \mathrm{~kg} / \mathrm{s}$ ) in Table 1, the flow field is expected to have the same velocity triangles. Consequently, the same flow mechanism of strut-compressor interaction is also expected. Hence, the operation point at 4600 RPM only is selected for numerical study in this paper. The compressor with eight inlet struts of $10 \mathrm{~mm}$ is taken as the reference designed as Case 1. Three more configurations are obtained by moving the struts axially, circumferentially, and reducing the strut diameter as shown in Table 3. The axial distance of " 3.5 R1" and "4.5 R1" means that the gap between the strut trailing 
edge and the rotor leading edge is 3.5 and 4.5 times the chord length of the rotor blade at midspan section. The relative circumferential position of the strut and stator " $0 S$ " is shown in Figure $2(\mathrm{c})$, while the " $0.5 \mathrm{~S}$ " represents the strut moving half of a stator pitch circumferentially.

The flow mechanism of the strut-compressor interaction is similar when the probe strut solidity is low, and increasing the number of struts will lead to increased strength of wake vortices shedding from the strut. Therefore, the change of strut number is not considered in Table 3. Meanwhile, reducing the axial distance or increasing the strut diameter will bring about the same blade vibration problem. Thus, only four cases in Table 3 are taken into account in the numerical investigation.

\section{Numerical Methods}

The numerical methods of fluid-structure interaction (FSI) include one-way fluid-structure interaction (OWI) and two-way fluid-structure interaction (TWI) methods. It is certain that the TWI method has higher accuracy in predicting the structural vibration, but consumes much more computing resource than the OWI does. Benra et al. [16] compared the feasibility of these two methods for predicting the structural vibration. If the shedding frequency of wake vortex from an upstream square cylinder was close to the natural frequency of the plate, the obtained frequency of the plate deflection was nearly the same for both methods and the plate displacement obtained using the OWI was smaller than that obtained using the TWI. Yin et al. [17] investigated the aerodynamic and mechanical performance of the last-stage long blade in a compressor. He found that the largest von Mises stress predicted by TWI is slightly smaller than that by OWI, but the same maximum stress location is predicted by the two methods. Despite all this, due to its lower time consumption, OWI is more widely used in solving engineering problems such as the blade resonance and compressor design $[18,19]$. According to the description of experimental results in Section 2, the blade resonance occurs under the influence of the inlet probe strut. Therefore, OWI is adopted to study the flow mechanism of compressor rotor blade vibration under different inlet probe configurations in this paper.

\subsection{Flow Calculation}

4.1.1. Meshing. The computational domain of the compressor with an inlet probe is shown in Figure 5. At the midspan section, the inlet boundary is 0.8 times the IGV chord length away from the IGV leading edge, and the outlet boundary is located at 2.7 times the R1 chord length downstream of the R1 trailing edge.

The grid is generated by NUMECA/Autogrid5 and IGG. The topology of blade/strut is $4 \mathrm{HO}$ for the main flow passage and is butterfly for the end gap. The near wall grid is refined to meet the requirement of the chosen turbulent model $\left(Y^{+}<1\right)$. In order to accurately capture the development of the strut shedding vortex, the wake region is refined, such as the green mesh in black box ( 5 million grids), the red mesh (8.84 million grids), and the orange mesh (16.5 million grids) in Figure 6(a). In addition, the grid is adjusted to the same distribution in the circumferential direction at the interfaces 2 and 3 to allow the strut shedding vortex to pass through smoothly. The grid distribution does not match at the interface 1 as shown in Figure 6(b), because no strut wake passes through there. The numbers of grid points of the domain S0 and domain R1 are 14.84 million and 16.5 million, respectively. A coarse grid with a total of 1.6 million grid points is used for the IGV domain, as it is to provide the inflow angle and turbulence to the inlet of the downstream domain. This results in 40.19 million grid points in total.

4.1.2. Solution Setup. The commercial CFD software CFX is used for the simulations to be presented in the paper. The second-order high-accuracy scheme is selected. Sørensen et al. [20] found that the results predicted by using the SSTDES turbulence model coupled with the $\gamma-\mathrm{Re}_{\theta}$ transition model are in good agreement with the experimental results when the Reynolds number $(\mathrm{Re})$ of the flow around cylinder is from 10 to $1 \times 10^{6}$. For the compressor studied in this paper, the Re of the flow around the strut is from $4.5 \times 10^{4}$ to $7 \times 10^{4}$. Therefore, the SST-DES turbulence model coupled with $\gamma-$ $\operatorname{Re}_{\theta}$ transition model is adopted for transient simulations.

According to the test conditions, the total pressure, the total temperature, and the axial flow angle are imposed at the domain inlet, and an average static pressure is set as the outflow boundary. The no-slip and adiabatic conditions are used for the solid wall. Rotational periodic boundary conditions are applied to the lateral surfaces of the computational domain. A mixing plane approach is used at the rotorstator interface in steady simulations, whereas a sliding interface technique is used in unsteady calculations. For unsteady calculation, the initial field is provided by the converged steady results and the physical time step is set to $\Delta t=5 \times$ $10^{-6} \mathrm{~s}$. The choice of time $\Delta t$ means that 30 physical time steps are required to pass through a rotor blade passage. The calculation results are considered to be converged when the flow field pressure fluctuation, the mass flow rate, and the efficiency have clear periodicity.

4.1.3. Validation. The flow mechanism of the strutcompressor interaction lies in accurate predictions of the wake vortex shedding from the strut. Therefore, the experimental results of the flow around a cylinder with $\mathrm{Re}=1 \times 10^{5}$ in Reference [21] are used to verify the numerical methods and grid distributions mentioned above. The comparison between the time-averaged results obtained by an unsteady calculation and the experimental data is shown in Figure 7. It can be seen that the predicted results of the friction coefficient distribution and separation position agree well with the experimental results, with only a small deviation of the peak friction coefficient. Therefore, the numerical calculation methods mentioned above are reliable for predicting the strut shedding vortex and the flow mechanism of strut-compressor interaction.

\subsection{Structural Calculation}

4.2.1. Meshing and Numerical Method. Because the geometry of the rotor blade disk is unknown, the rotor blade only is 


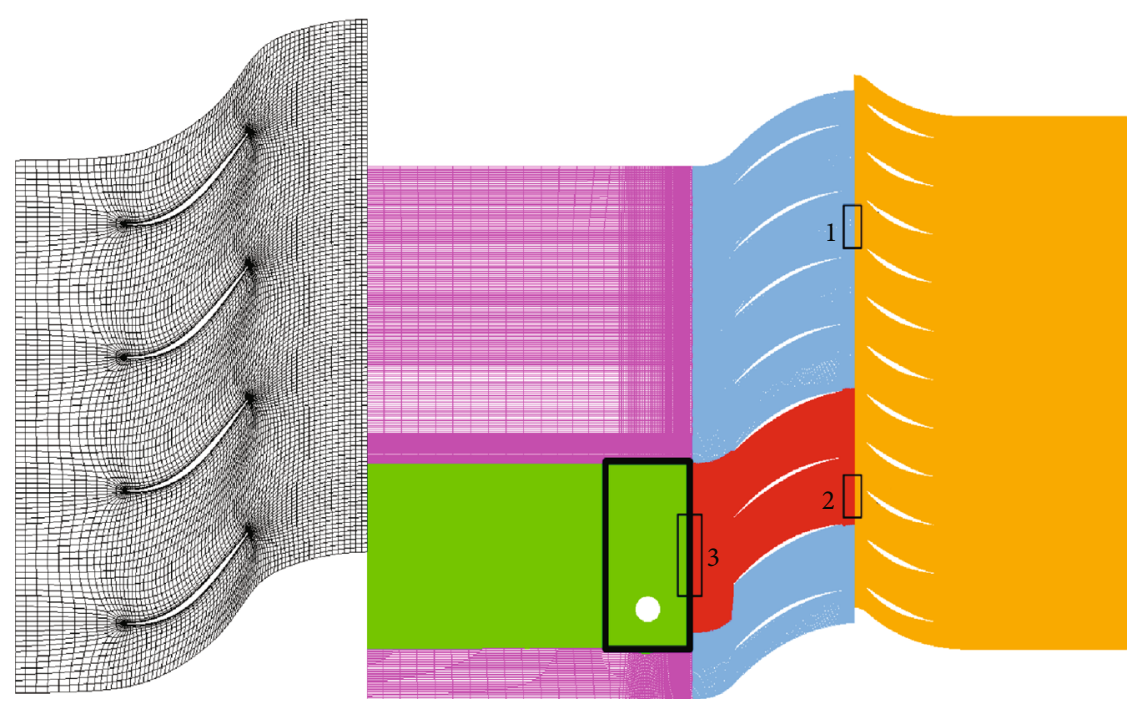

(a)
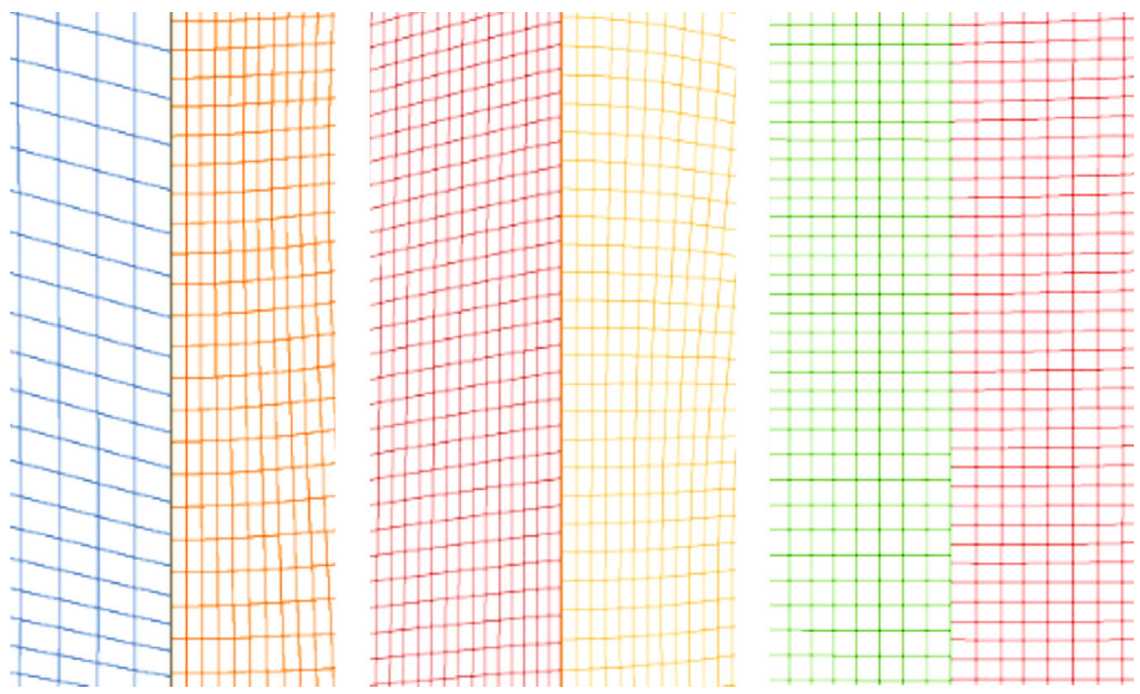

(b)

FIGURE 6: Schematic of the mesh: (a) blade to blade view at 50\% span and (b) close-up of the interface meshes (left: box 1, middle: box 2, and right: box 3).

analyzed for blade vibration. The commercial software ANSYS is used for blade meshing and vibration calculation. The finite element model of rotor blade is shown in Figure 8, with a total element number of 24017. A strain monitoring point $\mathrm{A}$ is set on the blade surface in Figure 8, corresponding to the first-order strain measurement position in Figure 3. The blade material is aluminum alloy 2A70 with the density of $2800 \mathrm{~kg} / \mathrm{m}^{3}$, Young's modulus of $7 \times 10^{10} \mathrm{~Pa}$, and Poisson's ratio of 0.33 . The degree of freedom at the bottom of the blade is set to 0 . The centrifugal load applied on the blade surface is obtained by a given blade rotation speed of 4600 RPM. The aerodynamic load on the blade surface is obtained by interpolating the pressure from a flow field analysis at the fluid-structure interface. The damping matrix is determined by Rayleigh damping, where the coefficients $\alpha$ and $\beta$ are calculated by the damping ratio of any two modes. In practical engineering, it is assumed that the modal damp- ing ratio of two vibration modes is equal and is given as 0.02 in empirical value. This assumption is adopted in the transient structural dynamic analysis by the Newmark direct integration method in this paper.

4.2.2. Validation. The procedures of transient structure dynamic calculation are as follows. The unsteady pressure, taken from the time interval of the compressor rotating four wheels after the convergence of flow simulation, is applied on the rotor blade surface firstly. Then, the transient structural dynamic calculation is carried out. The blade vibration signals, such as the strain and the displacement, are extracted and analyzed.

The strain signal obtained from the compressor test is used to verify the feasibility of the numerical methods for structural vibration analysis. For Case 1, the comparison of unsteady strain at point $\mathrm{A}$ on the rotor blade surface is shown 


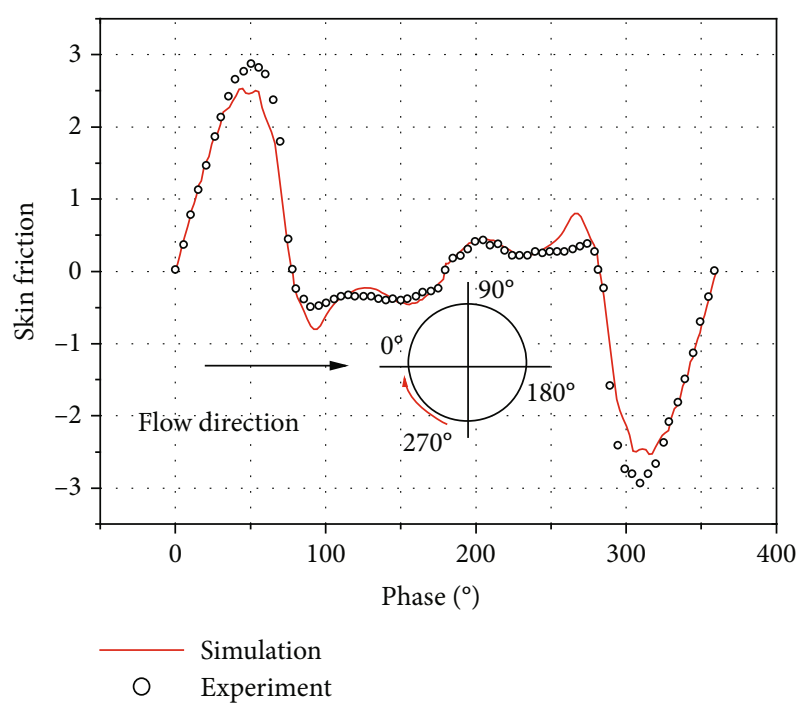

Figure 7: Results of skin friction coefficient of the cylinder flow.

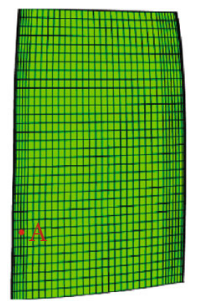

Figure 8: Finite element model of the rotor blade.

in Figure 9. Both results show that the strain gradually decays after reaching the peak value during the strut passing period, as shown by the red envelope line in Figure 9(a). The dominant frequency of strain in Figure 9 (b) is $906 \mathrm{~Hz}$, which is approximately equal to the calculated blade first-order natural frequency $908 \mathrm{~Hz}$ in Table 2. The calculation results suggest that the blade resonance occurs, agreeing with the conclusion of the test results in Section 2. Although the calculated maximum strain is $26 \%$ greater than the test data, the structural calculation method in this paper can be effectively used to analyze the relative change of blade vibration for different inlet probe configurations.

\section{Results and Discussions}

5.1. Rotor Blade Vibration. Corresponding to the cases in Table 3, Figures 10 and 11 show the time domain and frequency domain results of the strain at point $A$ and the rotor blade excitation force. In Figures 10(a) and 11(a), the peaks are enveloped by red lines, the maximum value is marked by red numbers, and the final peak-peak value represented by blue numbers is considered to be the strain amplitude and the excitation force amplitude. In Figures 10(b) and 11 (b), the rotor blade $1^{\text {st }}$ mode natural frequency $908 \mathrm{~Hz}$ is marked in red line, the strut passing frequency of $613 \mathrm{~Hz}$ and its harmonics are marked in blue lines, and the peak amplitude and corresponding frequency are labeled. In addition, the low frequency of $295 \mathrm{~Hz}$ obtained by subtract- ing $613 \mathrm{~Hz}$ from $908 \mathrm{~Hz}$ is shown by the purple line in Figure 11(b).

In Case 1, Case 2, and Case 3, with a strut diameter of $10 \mathrm{~mm}$, the strain amplitude gradually increases and finally maintains a high value in Figure 10(a). Meanwhile, the dominant frequency of the strain is close to the blade natural frequency in Figure 10(b). Therefore, the rotor blade resonance occurs under the influence of the upstream large-sized strut in the first three cases. Since the blade maximum strain in Case 2 and Case 3 is about $76 \%$ and $81 \%$ of that in Case 1, and the critical value of overstrain is $68 \%$ of the maximum strain found in the test at 4600 RPM, the strain gauge may issue the same over limit warning as in Case 1.

In contrast, the diameter of the strut is only $5 \mathrm{~mm}$ in Case 4. The blade maximum strain in Case 4 attenuates to a small value, which is only $16 \%$ of that in Case 1 . In addition, the frequency with the dominant amplitude is the fourth harmonic of the strut passing frequency, and the dominant amplitude in Case 4 is much smaller than that in the first three cases. Therefore, the blade vibrates safely in Case 4 .

Then, the excitation force causing the blade vibration is analyzed. In Figure 11(b), the excitation force component with a frequency close to $908 \mathrm{~Hz}$ cannot be ignored in Case 1, Case 2, and Case 3, but the opposite is in Case 4. In Figure 11(a), both the amplitude and the maximum value of the excitation force in Case 1, Case 2, and Case 3 are larger than that in Case 4. That is, in Case 1-Case 3 with a largesized strut, the rotor blade is subjected to an excitation force with a frequency just equal to its $1^{\text {st }}$ order natural frequency and a large amplitude. In Case 4, the rotor excitation force is small. According to the resonance theory, it happens if the excitation force frequency is close to the natural frequency and the energy gained by the blade is greater than that dissipated. The absorbed energy is mainly determined by the amplitude of excitation force. Therefore, the frequency and amplitude of the excitation force induced by the upstream strut play an important role in the blade vibration.

In Figure 11(b), the frequencies close to $908 \mathrm{~Hz}$ and other higher ones can be obtained by superimposing low frequencies close to $295 \mathrm{~Hz}$ and $613 \mathrm{~Hz}$. The frequency close to $295 \mathrm{~Hz}$ can only be found in Case 1-Case 3 with the largesized strut, so it is named as the strut-wake-induced frequency in this paper. Because the $613 \mathrm{~Hz}$ is determined by the rotation speed and the number of probes, the estimation of another frequency $295 \mathrm{~Hz}$ is essential for blade resonance.

In Figure 11(a), the amplitude of the excitation force depends on the strut configuration. Once the compressor flow field is affected by the upstream wakes of foreign struts, the excitation force on the rotor blade will change accordingly. Therefore, in order to minimize the amplitude of the excitation force, it is critical to assess the strength of the strut wake for different strut configurations.

\subsection{Flow Mechanism of the Strut-Compressor Interaction}

5.2.1. Spectral Analysis of the Excitation Force. The source and estimation method of the strut-wake-induced frequency are analyzed below, taking Case 1 as the example. Figure 12 shows the spectrum results of unsteady pressure detected at 


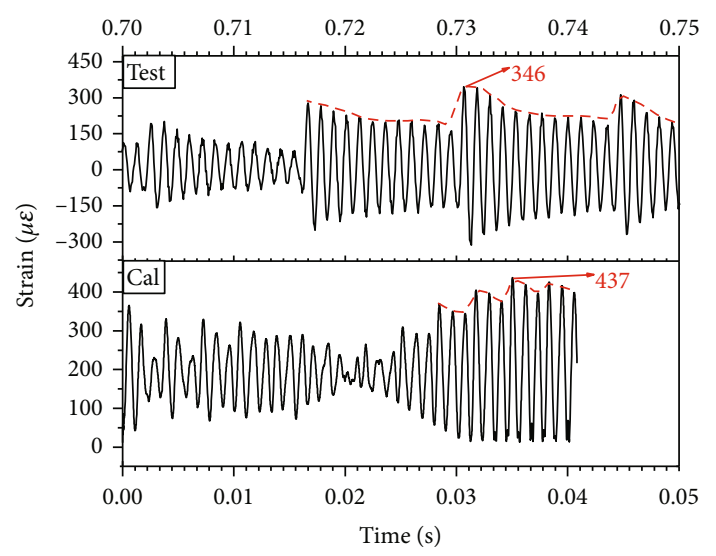

(a)

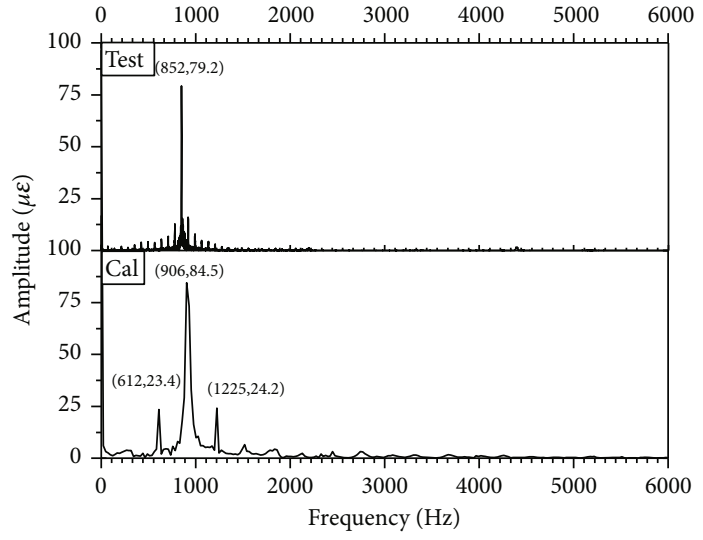

(b)

Figure 9: Comparison of experimental and numerical results of the strain at point A in Case 1: (a) time domain and (b) frequency domain.

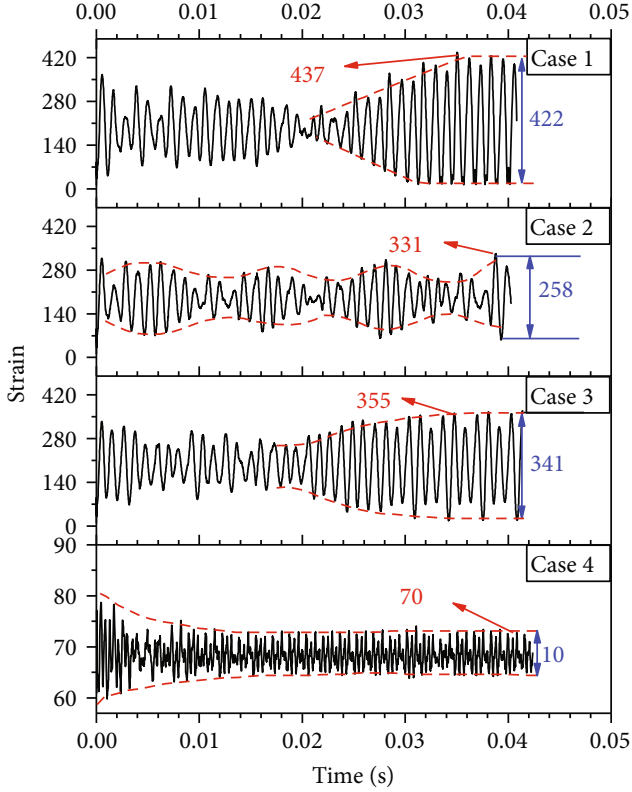

(a)

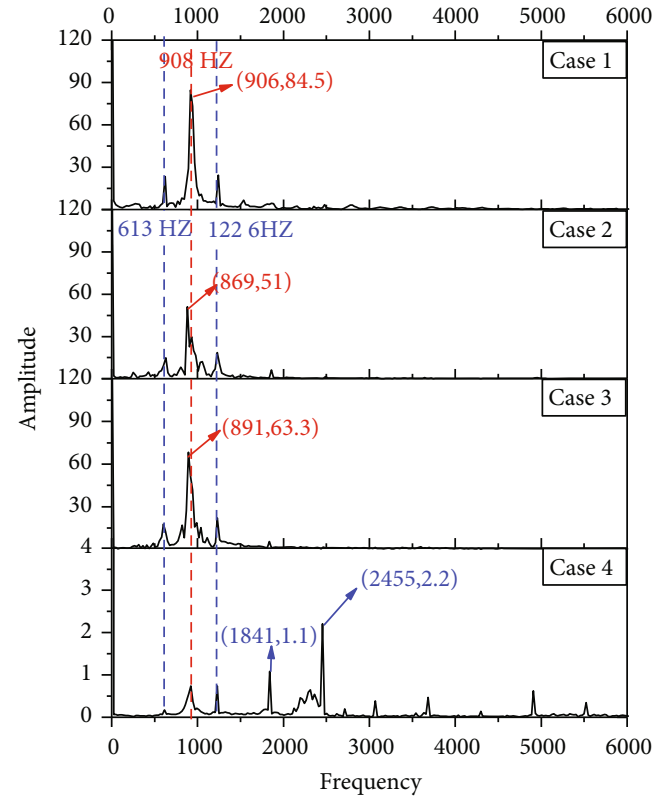

(b)

Figure 10: The strain at point A for different strut configurations: (a) time domain and (b) frequency domain.

point $\mathrm{PA}$ and $\mathrm{PB}$. The two points $\mathrm{PA}$ and $\mathrm{PB}$ are at $58 \%$ span section. The point PA is in the wake zone and is 1.5 times the strut diameter downstream of the strut trailing edge, and the point $\mathrm{PB}$ is in the stator wake zone and is $6 \mathrm{~mm}$ away from the stator trailing edge. It can be seen that the frequency of the wake vortex shedding from the strut is $2125 \mathrm{~Hz}$, which is labeled as $f_{\text {shedding }}$. And all the determined frequencies of the strut and the blade passing mentioned above are greater than $613 \mathrm{~Hz}$. Therefore, the origin of low frequency close to $295 \mathrm{~Hz}$ in Figure 11 should be obtained by further exploring the unsteady flow mechanism of the strut-compressor interaction.

Figure 13 shows the time history of the inflow attack angle measured by a numerical probe mounted on the rotating hub upstream of the rotor blade over 44 the rotor blade passing periods. At the midspan section, the axial distance between the numerical probe and the rotor blade leading edge is $1 \mathrm{~mm}$, and the circumferential distance along the rotation direction is $2 \mathrm{~mm}$. In Figure $13, h / H$ is the relative height of the blade, and $N_{\mathrm{NRP}}$ represents the number of rotor blade passing periods.

As shown in Figure 13, the positive attack angle has four peaks, that is, one peak appears every $11 N_{\mathrm{NRP}}$. The ratio of the strut count to the rotor blade count is $1: 11$, so the peak attack angle is caused by the upstream strut. Large fluctuations in the attack angle lead to the large fluctuations in blade load and the corresponding excitation force. It is the reason why the frequency of the excitation force with the dominant amplitude is close to $613 \mathrm{~Hz}$ in Figure 11(b).

In addition, in Figure 13, the peak positive attack angles appear approximately once every $22 N_{\mathrm{NRP}}$, that is the consequence of asynchronization of the rotor inlet attack angle and 


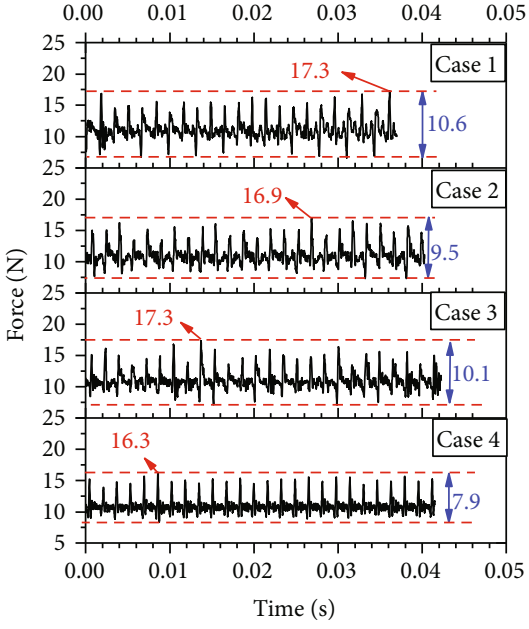

(a)

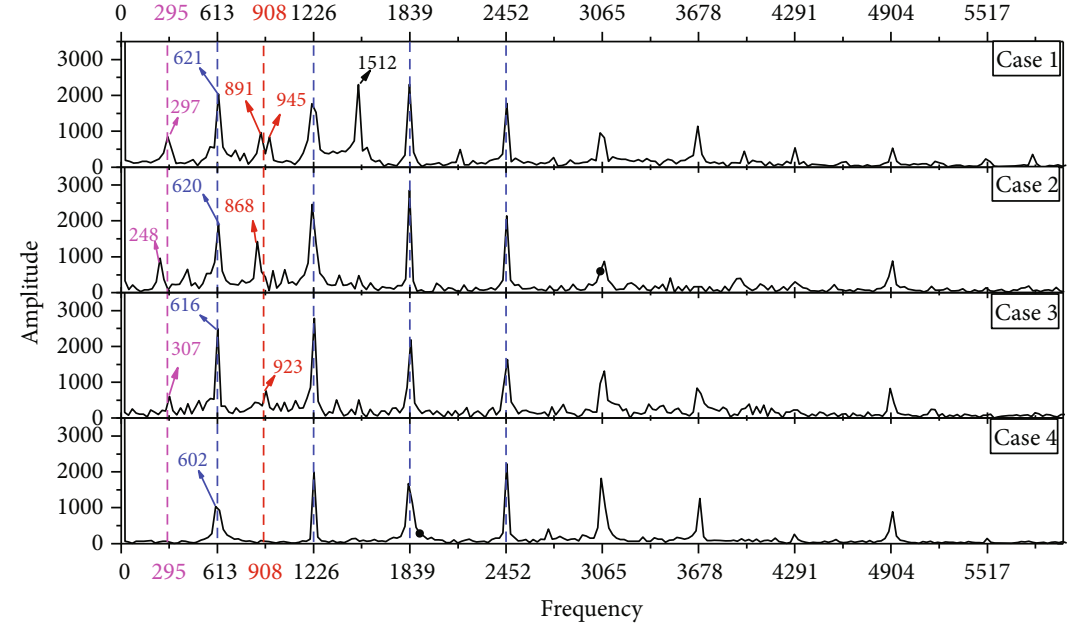

(b)

Figure 11: The rotor blade excitation force for different strut configurations: (a) time domain and (b) frequency domain.

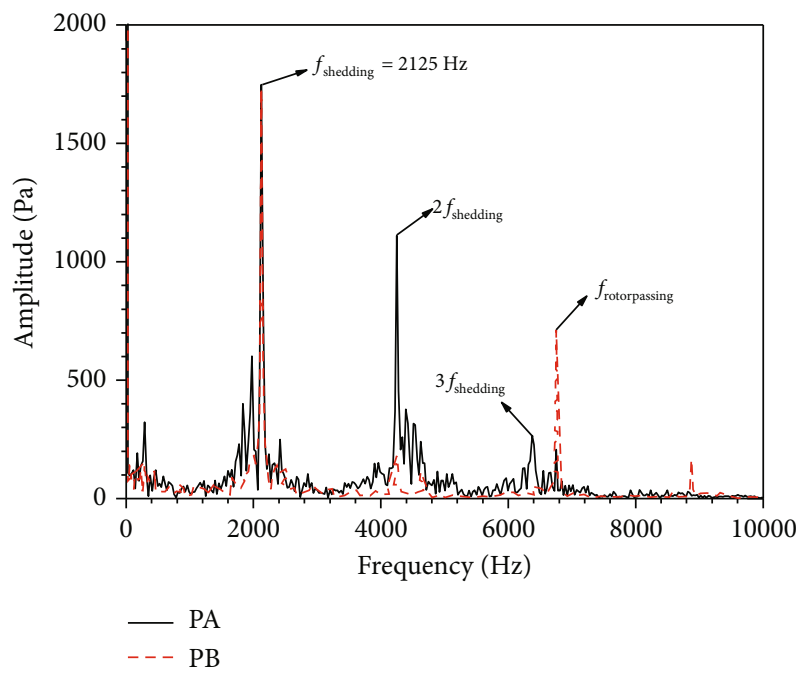

FIGURE 12: FFT results of the unsteady pressure at point PA and PB in Case 1.

the upstream strut wake vortex. The instantaneous vorticity contours of the midspan section at $t=t_{0}, t_{0}+T_{\text {rotorpassing, }}, t_{0}+$ $11 T_{\text {rotorpassing }}$, and $t_{0}+22 T_{\text {rotorpassing }}$ are shown in Figure 14. It can be seen that the flow behaves similarity only at $t=t_{0}$ and $t=t_{0}+22 T_{\text {rotorpassing, }}$ although the relative positions of the strut, the stator, and the rotor are the same at these four instants. Since the rotor blade load is the same for the similar flow field, the excitation force with a low frequency of $306.7 \mathrm{~Hz}$ can be calculated from $22 T_{\text {rotorpassing. Like the }}$ strut-wake-induced frequency around $295 \mathrm{~Hz}$ in Figure 11, $306.7 \mathrm{~Hz}$ can also be regarded as the strut-wake-induced frequency.

Look closely at Figures 14(a)-14(c), the difference of nonsimilar flow is caused by the strut shedding vortex, which has a different phase at the strut trailing edge and at the rotor

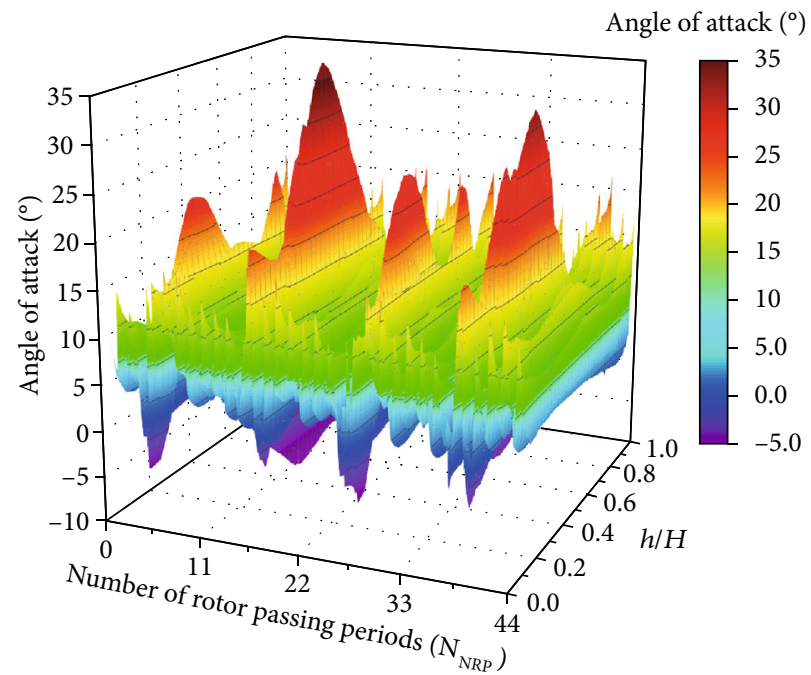

FIGURE 13: Contours of the rotor blade inflow attack angle in Case 1.

leading edge. Therefore, the strut-wake-induced frequency can be estimated by flow similarity.

$$
\begin{aligned}
T & =m \times T_{\text {rotorpassing }}=n \times T_{\text {shedding }}=k \times T_{\text {transport }} \\
& =p \times T_{\text {probepassing }}
\end{aligned}
$$

where $m, n, k$, and $p$ are all positive integers. $T_{\text {shedding }}$ is the period of wake vortex shedding from the strut, and $T_{\text {transport }}$ is the time required for the vortex to transport from the strut trailing edge to the rotor leading edge.

From Equation (1), the strut-wake-induced frequency is $1 / p$ times of the strut passing frequency.

From Case 1 to Case $3, T_{\text {shedding }}$ is approximately the same for the strut with $10 \mathrm{~mm}$ in diameter, and $T_{\text {transport }}$ is almost equal for the little change of axial distance between the strut and the rotor blade. $T_{\text {probepassing }}$ and $T_{\text {rotorpassing }}$ 


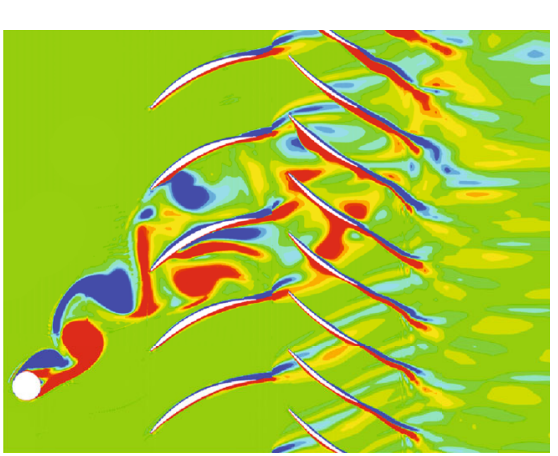

(a)

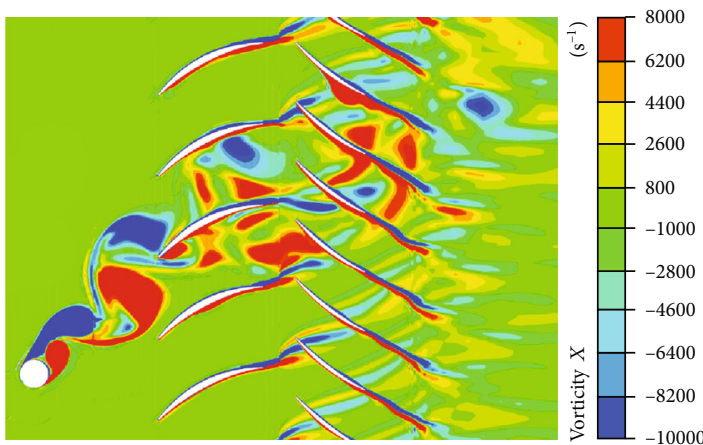

(c)

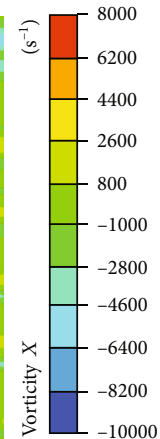

$-10000$
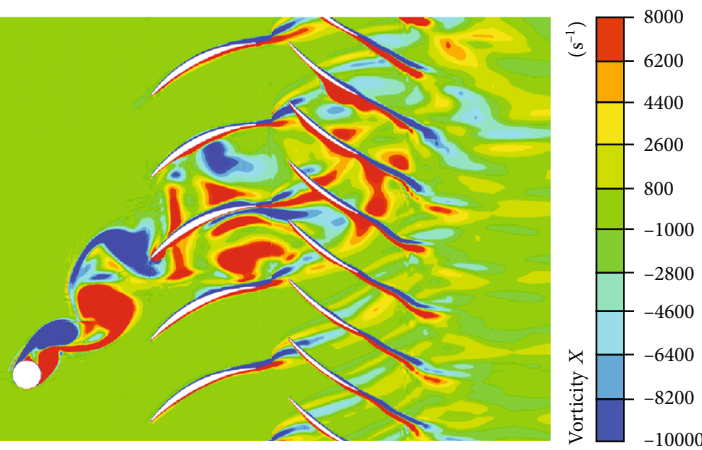

(b)

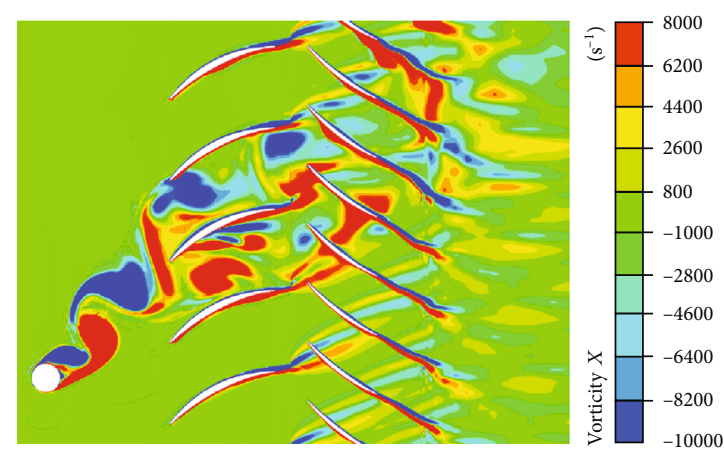

(d)

Figure 14: Instantaneous vorticity contours for Case 1: (a) $t=t_{0}$;

; (b) $t=t_{0}+T_{\text {rotorpassing }}$ (c) $t=t_{0}+11 T_{\text {rotorpassing; }}$;

; (d) $t=t_{0}+22 T_{\text {rotorpassing. }}$

remain constants for the unchanged rotating speed and the number of the strut and rotor blade. Thus, the strut-wakeinduced frequency predicted by Equation (1) keeps almost unchanged, which is verified in Figure 11.

5.2.2. Strength Analysis of the Strut Wake Vortex. Because of the coupling of velocity and pressure in the Navier-Stokes equation, the pressure fluctuation can also be used to describe the strength of the wake vortices which are shed from the strut and transported downstream. In this paper, the wake vortex strength is defined as the root mean square of the pressure $P_{\text {rms }}=\sqrt{\sum_{i=1}^{N}\left(P-P_{\text {ave }}\right)^{2} /(N-1)}$. Taken the wake strength at point PA in Case 1 as the reference, the dimensionless wake strength $\bar{P}_{\text {rms }}$ is calculated, as shown in Figure 15. It can be seen that the strut-wake strength changes with the strut configuration.

The changes of wake strength at PA are analyzed firstly, which can be explained by the strut-stator interaction. The strut wake affected by the downstream stator can be analogous to that of the cylinders arranged in series or crossed. Zdravkovich [22] found that there is a critical distance of 45 times diameter of cylinder in series arrangement, below which the interaction of adjacent cylinders cannot be ignored, because it causes a greater drag coefficient. According to Wu's [23] study, the cylinder wake strength is proportional to the drag coefficient. In Case 1, at the midspan section, the small streamwise distance between the strut and the stator results in the nonignorable strut-stator interaction. Since the drag coefficient of the cylinders arranged in

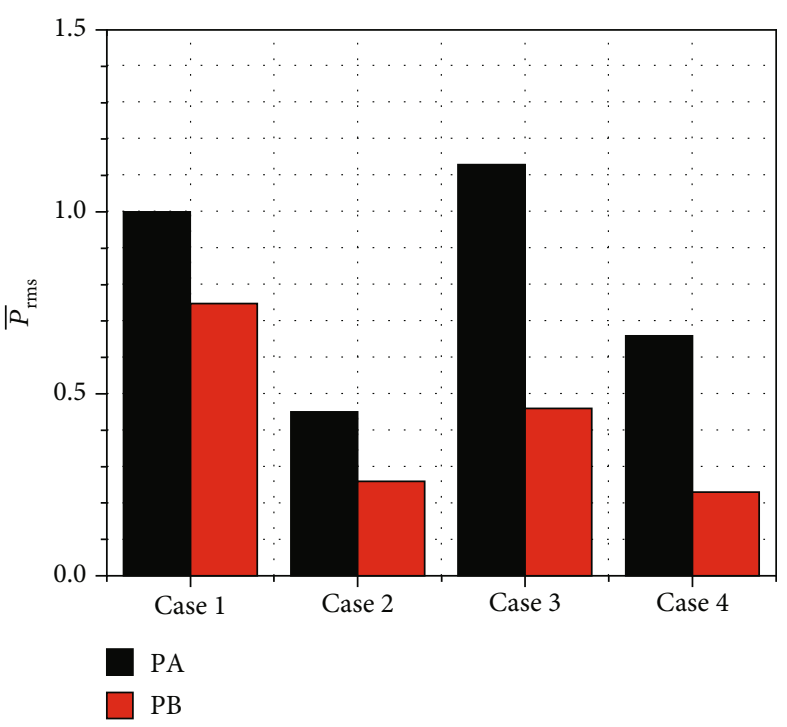

FIgURe 15: Strut-wake strength at point PA and point $\mathrm{PB}$ for different strut configurations.

crossed is smaller than that in series [22], the configuration changes of the strut and stator reduce the strut-wake strength significantly from Case 1 to Case 2. In addition, the shorter the dimensionless distance, the stronger the strut-stator interaction, where the dimensionless distance is defined as the ratio of the distance between the strut and stator to the strut diameter. The decrease of the strut diameter from 
$10 \mathrm{~mm}$ to $5 \mathrm{~mm}$ at the same strut location leads to an increase in dimensionless distance. Thus, the wake strength in Case 4 is weaker than that in Case 1 but stronger than that in Case 2. And the strut in Case 3 is closer to the middle of the transition duct, so the strut-wake strength is affected more by the adverse pressure gradient of S-type transition duct rather than the reduction of the dimensionless distance. This is the reason why the strut-wake strength in Case 3 is greater than that in Case 1.

Then, the wake strength at PB is discussed. It is determined by the initial wake strength at PA and the wake decay rate from the strut to the stator. The larger the dimensionless distance, the greater the decay rate of wake vortex. For dimensionless distance, the largest value is in Case 4 , the second largest is in Case 3, and the smallest is in Cases 1 and 2. So although the strut-wake strength at PA in Case 4 is larger than that in Case 2, the wake strength at PB is opposite. The same conclusion is true for Case 3 and Case 1. Therefore, the final wake strength at PB is sorted in order as Case 1, Case 3, Case 2, and Case 4, which is consistent with the strain in Figure 10.

Through the analyses above, reasonable probe configuration can reduce the amplitude of blade excitation force. Both reducing the adverse pressure gradient of the transition duct where the strut is located and making the strut wake to pass through the midpassage of downstream stator are conducive to decreasing the strut-wake strength. Enlarging the gap between the strut and the stator, and reducing the strut diameter are beneficial to reduce the strut-wake strength and increase the wake dissipation. It is helpful to guide test engineers to choose the probe strut configuration in compressor tests.

\section{Conclusions}

In order to explain the overstrain problem of the first-stage rotor blade caused by the inlet probe in the compressor performance tests, the one-way fluid-structure coupling simulation method was adopted. The inlet probe simplified as a strut with a diameter of $10 \mathrm{~mm}$; the inlet stator and the first-stage rotor in the test compressor were selected as the research objects. Numerical calculation is carried out to study the effect of strut configuration on the strain of rotor blade vibration and the flow mechanism of strut-compressor interaction. The conclusions are as follows:

(1) The frequency and the amplitude of the excitation force are two important factors that affect rotor blade vibration. When one of the excitation force frequencies is close to a natural frequency of the rotor, the blade resonates, and the amplitude of blade strain varies with the amplitude of the excitation force

(2) The frequencies of the excitation force are affected by the interaction between the strut and the compressor. Besides the strut passing frequency, there is a lower frequency activated by large-sized strut in the excitation force, which is named as the strut-wake-induced frequency. The wakeinduced frequency is related to the passing frequency of the strut and the rotor blade, the wake vortex shedding frequency of the strut, and the time for the strut wake to be transported to the rotor leading edge. Based on the frequency correlation, a prediction model of the wake-induced frequency is established in the paper

(3) The amplitude of the excitation force is related to the probe configuration. Adjusting the strut-stator arrangement to decrease the strut-wake strength and increasing the dimensionless distance between the strut and stator to increase the wake decay rate are two effective ways for reducing the amplitude of the excitation force

(4) To avoid the occurrence of rotor-blade overstrain in compressor performance tests, the inlet probe should be designed with the strut diameter as small as possible under the constraints of probe processing, structural strength. Meanwhile, on the premise of meeting the requirements of probe geometric installing space and the compressor performance test, the probe needs to be placed far upstream of the rotor blade with the strut wake passing through the midpassage of the inlet stator
Abbreviations
RPM: Revolutions per minute
IGV: Inlet guide vane
FSI: $\quad$ Fluid-structure interaction
OWI: One-way fluid-structure interaction
TWI: Two-way fluid-structure interaction
FFT: $\quad$ Fast Fourier transform
$f_{\text {shedding: }}$ The strut-wake shedding frequency
$N_{\text {NRP }}$ : The number of the rotor blade passing periods
$P_{\mathrm{rms}}$ : $\quad$ Root mean square of the pressure.

\section{Data Availability}

The data used to support the findings of this study are available from the corresponding author upon request.

\section{Conflicts of Interest}

The authors declare no conflict of interest.

\section{Authors' Contributions}

Yang contributed the central idea, analyzed most of the data, and wrote the initial draft. The remaining authors contributed to refining the ideas, carrying out additional analyses, and finalizing the paper. All authors read and approved the manuscript.

\section{Acknowledgments}

This work was funded by the Special Scientific Research Project for Civil Aircraft (Grant No. MJ-2016-J-96). And the work was funded by the National Natural Science Foundation of China (Grant No. 51406082).

\section{References}

[1] H. Ma, S. Li, and W. Wei, "Effects of probe support on the flow field of a low-speed axial compressor," Journal of Thermal Science, vol. 23, no. 2, pp. 120-126, 2014. 
[2] R. Yang, J. Zhao, and H. Xiang, "Effects of inlet probe support on aerodynamic performance of 1.5-stage compressor," Journal of Propulsion Technology, vol. 38, pp. 1038-1046, 2017.

[3] H. Ma and L. Xiang, "Numerical investigation of effects of probe support on performance and flow field of compressor rotor," Journal of Propulsion Technology, vol. 37, pp. 22882295, 2016.

[4] J. Lepicovsky, "Effects of a Rotating Aerodynamic Probe on the Flow Field of a Compressor Rotor," Tech. Rep. NASA/CR2008-215215, 2008.

[5] H. Xiang, N. Ge, and M. Hou, "An experimental investigation of the effects of upstream probe disturbance on compressor cascade performance," Proceedings of the Institution of Mechanical Engineers, Part G: Journal of Aerospace Engineering, vol. 231, pp. 1248-1257, 2017.

[6] C. Teruna, D. Ragni, F. Avallone, and D. Casalino, "A rodlinear cascade model for emulating rotor-stator interaction noise in turbofans: a numerical study," Aerospace Science and Technology, vol. 90, pp. 275-288, 2019.

[7] R. M. C. So, I. Jadic, and M. P. Mignolet, "Fluid-structure resonance produced by oncoming alternating vortices," Journal of Fluids and Structures, vol. 13, pp. 519-548, 1999.

[8] R. C. K. Leung and R. M. C. So, "Noise generation of bladevortex resonance," Journal of Sound and Vibration, vol. 245, no. 2, pp. 217-237, 2001.

[9] P. Barreca, L. Pinelli, F. Vanti, and A. Arnone, "Aeroelastic investigation of a transonic compressor rotor with multi-row effects," Energy Procedia, vol. 148, pp. 58-65, 2018.

[10] C. Sanders, M. Terstegen, P. Jeschke, H. Schoenenborn, and J. P. Heners, "Rotor-stator interactions in a 2.5-stage axial compressor-part II: impact of aerodynamic modeling on forced response," Journal of Turbomachinery, vol. 141, no. 10, article 101008, 2019.

[11] Z. Yun, Q. Gao, and H. Yang, "Aeroelastic vibration analysis of a 1.5 stage compressor," Propulsion and Power Research, vol. 9, pp. 26-36, 2020.

[12] H. Taher and S. G. Mohamed, "Numerical investigation of compressor blades deformation during stall development into surge," Journal of Propulsion and Power, vol. 33, no. 5, pp. 1074-1086, 2017.

[13] F. Liang, Z. Xie, and A. Xia, "Aeroelastic simulation of the first 1.5-stage aeroengine fan at rotating stall," Chinese Journal of Aeronautics, vol. 33, no. 2, pp. 529-549, 2020.

[14] P. S. Murari and M. L. George, Blade Design and Analysis for Steam Turbines, McGraw-Hill Education, USA, 2011.

[15] M. M. Zdravkovich, "Review of interference-induced oscillations in flow past two parallel circular cylinders in various arrangements," Journal of Wind Engineering and Industrial Aerodynamics, vol. 28, no. 1-3, pp. 183-199, 1988.

[16] F. K. Benra, H. J. Dohmen, J. Pei, S. Schuster, and B. Wan, “A comparison of one-way and two-way coupling methods for numerical analysis of fluid-structure interactions," Journal of Applied Mathematics, vol. 2011, 16 pages, 2011.

[17] M. Yin, J. Li, L. Song, and Z. Feng, "Numerical investigations of the long blade performance using RANS solution and FEA method coupled with one-way and two-way fluid-structure interaction models," in Volume 2: I\&C, Digital Controls, and Influence of Human Factors; Plant Construction Issues and Supply Chain Management; Plant Operations, Maintenance, Aging Management, Reliability and Performance; Renewable Energy Systems: Solar, Wind, Hydro and Geothermal; Risk
Management, Safety and Cyber Security; Steam Turbine-Generators, Electric Generators, Transformers, Switchgear, and Electric BOP and Auxiliaries; Student Competition; Thermal Hydraulics and Computational Fluid Dynamics, Charlotte, North Carolina, USA, 2017.

[18] X. Zheng, C. Ding, and Y. Zhang, "Influence of different loads on the stresses of multistage axial compressor rotors," Proceedings of the Institution of Mechanical Engineers, Part G: Journal of Aerospace Engineering, vol. 231, pp. 787-798, 2017.

[19] T. R. Jebieshia, S. K. Raman, and H. D. Kim, "Aerodynamic and structural characteristics of a centrifugal compressor impeller," Applied Sciences, vol. 9, no. 16, article 3416, 2019.

[20] N. N. Sørensen, A. Bechmann, and F. Zahle, "3D CFD computations of transitional flows using DES and a correlation based transition model," Wind Energy, vol. 14, no. 1, pp. 77-90, 2011.

[21] E. Achenbach, "Distribution of local pressure and skin friction around a circular cylinder in cross-flow up to $\mathrm{Re}=5 \times 10^{6}$," Journal of Fluid Mechanics, vol. 34, pp. 625-639, 2006.

[22] M. M. Zdravkovich, Flow around Circular Cylinders Vol 2: Applications, Oxford University Press, UK, 2003.

[23] J. C. Wu, Elements of Vorticity Aerodynamics, Springer, Germany, 2005. 